\title{
A User Selection Algorithm Based On the Combination of Block Diagonalization and SLNR Maximization
}

\author{
Weihong Fu, Cheng Wang, Nai'an Liu, Qingliang Kong, Weixin Tian \\ State Key Lab. Of Integrated Service Networks, Xidian University \\ Xi'an, China \\ e-mail: whfu@mail.xidian.edu.cn
}

\begin{abstract}
In this paper, a new precoding scheme is proposed based on the combination of Block Diagonalization (BD) and SLNR (Signal Leakage Noise Ratio) maximization. Then a new user selection algorithm is proposed based on the joint precoding scheme. BD precoding will cause performance loss in the single antenna terminals when the number of terminal antenna is inconsistent. The algorithm we proposed can overcome the drawback by using the maximum SLNR for single-antenna users and BD precoding for multi-antenna users respectively. Simulation results show that the proposed algorithm will enhance the system sum-rate performance significantly when SNR (Signal Noise Ratio) over 5dB. The performance improves by $30 \%$ when SNR reaches $20 \mathrm{~dB}$.
\end{abstract}

Keywords- Block Diagonalization (BD); Signal Leakage Noise Ratio (SLNR); User Selection; joint precoding

\section{INTRODUCTION}

Multiple input multiple output (MIMO) systems can effectively improve the capacity and transmission rate of wireless communication systems [1]. In multiuser MIMOBC (Multiple-Input Multiple-Output Broadcasting) system, the interference between users must be considered. Dirty Paper Coding (DPC) is the best precoding algorithm can completely eliminate the interference between users and reach the capacity of the system [4-5]. But it is difficult to implement in practical systems due to the high computational burden of successive encodings and decodings, especially when the number of users is large. Block Diagonalization (BD) whose basic idea is decomposing the multi-user downlink channel into several independent and parallel single-user MIMO downlink channels has greatly reduced the complexity compared to DPC. In the case that channel state information is completely known, the signal of each user is pre-processed at the transmitter using a precoding matrix that lies in the null space of other users' channel matrices. In this way the interference that this user represents for the others is set to zero. There exist some user selection algorithms using BD precoding [6-7]. However, non of them take into account the situation that some users have more than one antennas while some have only one in a system, in this case, the performance of single antenna users will reduce the overall system performance by using BD precoding [8]. However, with the development of science and technology, the case that one terminal configures more than one antenna will be more and more while the single antenna users still exist in the system at the same time. So simply using BD precoding is no longer applicable. The precoding scheme based on maximum SLNR (Signal Leakage Noise Ratio) can achieve better performance when the terminal have single antenna. So a new precoding scheme is proposed based on combination of BD and SLNR maximization. In multi-user MIMO system, the number of users a system can support simultaneously is subjected to the restriction on the number of base station antennas. So we should choose the best group from all users to communicate. The optimal user selection algorithm is to try all the combinations of users to find the user group that can achieve the multiuser diversity. However, the calculation amount of the optimal algorithm is too large to implement. We propose a new user selection algorithm based on the combination of $\mathrm{BD}$ and maximum SLNR to select user group for communication.

\section{PRECODING TECHNIQUES}

\section{A. Block Diagonalization (BD)}

$\mathrm{BD}$ is a linear precoding scheme that can be applied to multi-user MIMO downlink system and decomposing the multi-user downlink channel into several independent and parallel single-user MIMO downlink channels [9]. The interference between users can be eliminated by choosing the $j$ th user's precoding matrix $\mathbf{M}_{j}$ in the null space of all other users' channel matrices, satisfy the following formula:

$$
\mathbf{H}_{i} \mathbf{M}_{j}=\mathbf{0}(i \neq j)
$$

Where $\mathbf{H}_{i}$ is the $i$ th user's channel matrix.

We define $\tilde{\mathbf{H}}_{j}$ as

$$
\tilde{\mathbf{H}}_{j}=\left[\mathbf{H}_{1}^{\mathrm{H}} \ldots . \mathbf{H}_{j-1}^{\mathrm{H}} \mathbf{H}_{j+1}^{\mathrm{H}} \ldots . \mathbf{H}_{K}^{\mathrm{H}}\right]^{\mathrm{H}}
$$

$\tilde{\mathbf{H}}_{j}$ is called the complement matrix of $\mathbf{H}_{j}$. According to equation (1), we have $\tilde{\mathbf{H}}_{j} \mathbf{M}_{j}=\mathbf{0}$. 
$\tilde{\mathbf{H}}_{j}$ is subjected to Singular Value Decomposition (SVD) as follows.

$$
\tilde{\mathbf{H}}_{j}=\tilde{\mathbf{U}}_{j} \tilde{\Sigma}_{j}\left[\tilde{\mathbf{V}}_{j}^{(1)} \tilde{\mathbf{V}}_{j}^{(0)}\right]^{\mathrm{H}}
$$

We choose the last right singular vectors $\tilde{\mathbf{V}}_{j}^{(0)}$ which form an orthogonal basis for the null space of $\tilde{\mathbf{H}}_{j}$. The equivalent null space channel matrix of user $j$ is identified as $\mathbf{H}_{j} \tilde{\mathbf{V}}_{j}^{(0)}$. Define the SVD

$$
\mathbf{H}_{j} \tilde{\mathbf{V}}_{j}^{(0)}=\mathbf{U}_{j} \boldsymbol{\Sigma}_{j}\left[\mathbf{V}_{j}^{(1)} \mathbf{V}_{j}^{(0)}\right]^{\mathrm{H}}
$$

Then the precoding matrix for user $j$ is

$$
\mathbf{M}_{j}=\tilde{\mathbf{V}}_{j}^{(0)} \mathbf{V}_{j}^{(0)}
$$

And $\mathbf{V}_{j}^{(1)}$ can be used as receive matrix.

\section{B. Maximum SLNR Precoding}

Putting all users as a whole, we always hope the interference generated by user $i$ to other users can be minimal while the energy of user $i$ reaches the maximum at the same time. So there have the maximum SLNR criterion [10-11]. The SLNR is defined as

$$
\begin{aligned}
& \operatorname{SLNR}_{i}=\frac{\left\|\mathbf{h}_{i} \mathbf{w}_{i}\right\|^{2}}{\boldsymbol{\sigma}_{i}^{2}+\sum_{k=1, k \neq i}^{K}\left\|\mathbf{h}_{k} \mathbf{w}_{i}\right\|^{2}} i=1,2, \ldots, K \\
& \text { Where } \sum_{k=1, k \neq i}^{K}\left\|\mathbf{h}_{k} \mathbf{w}_{i}\right\|^{2} \text { is the interference generated by }
\end{aligned}
$$
user $i$ to other users, $\left\|\mathbf{h}_{i} \mathbf{w}_{i}\right\|^{2}$ is the energy of user $i$.

We can find that each user's SLNR only relate with its own precoding matrix. Therefore, we can be relatively easy to choose the best precoding matrix by maximizing SLNR, which can be expressed as

$$
\begin{aligned}
& \mathbf{w}_{i}^{\circ}=\arg \max _{\mathbf{w}_{i} \in C^{N_{t} \times 1}} \frac{\left\|\mathbf{h}_{i} \mathbf{w}_{i}\right\|^{2}}{\boldsymbol{\sigma}_{i}{ }^{2}+\sum_{k=1, k \neq i}^{K}\left\|\mathbf{h}_{k} \mathbf{w}_{i}\right\|^{2}} \\
& =\arg \max _{\mathbf{w}_{i} \in C^{N_{1} \times 1}} \frac{\mathbf{w}_{i}^{*} \mathbf{h}_{i}^{*} \mathbf{h}_{i} \mathbf{w}_{i}}{\mathbf{w}_{i}^{*}\left(\boldsymbol{\sigma}_{i}^{2} \mathbf{I}+\overline{\mathbf{H}}_{i}^{*} \overline{\mathbf{H}}_{i}\right) \mathbf{w}_{i}}
\end{aligned}
$$

Where $\overline{\mathbf{H}}_{i}=\left[\mathbf{h}_{1} \ldots \mathbf{h}_{i-1} \mathbf{h}_{i+1} \ldots \mathbf{h}_{K}\right]$.
According to the literature [12], we know that the optimal solution of equation (7) is the largest eigenvalue eigenvector of $\left(\boldsymbol{\sigma}_{i}^{2} \mathbf{I}+\overline{\mathbf{H}}_{i}^{*} \overline{\mathbf{H}}_{i}\right)^{-1} \mathbf{h}_{i}^{*} \mathbf{h}_{i}$, that is

$$
\mathbf{w}_{i}^{\text {o }} \propto \text { eigenvector }\left(\left(\boldsymbol{\sigma}_{i}^{2} \mathbf{I}+\overline{\mathbf{H}}_{i}^{*} \overline{\mathbf{H}}_{i}\right)^{-1} \mathbf{h}_{i}^{*} \mathbf{h}_{i}\right)
$$

\section{Combination of BD and maximum SLNR}

The performance of single antenna users will reduce the overall system performance by using BD precoding when the number of terminal antenna is inconsistent. In order to improve the overall system performance, especially the performance of single antenna users, we propose the precoding scheme based on combination of $\mathrm{BD}$ and SLNR maximization. The main idea of this method is as follows.

1) The precoding matrices for multiple antenna users are chosen to lie in the null space of the channel matrices of the other users including the single antenna users. In this way the equivalent channel for the single antenna users looks as if there were no multiple antenna users.

2) The precoding matrices for single antenna users are chosen by the maximum SLNR criterion.

\section{USER SELECTION ALGORITHM}

\section{A. System model}

Consider a single-cell MIMO BC with a single base station and $N$ user terminals. The base station is equipped with $M$ transmit antennas and the $k$ th user terminal with $N_{k} \quad$ receive antennas. We assume that $N \geq M \geq N_{k}$. We use a simple channel model where the channel gain from a transmit antenna to a user is described by a zero-mean circularly symmetric complex Gaussian random variable. Suppose the Channel State Information (CSI) is completely known by the transmitter. The signal received by user $k$ may be written as:

$$
\mathbf{y}_{k}=\mathbf{H}_{k} \mathbf{W}_{k} \mathbf{x}_{k}+\sum_{j=1, j \neq k}^{K} \mathbf{H}_{k} \mathbf{W}_{j} \mathbf{x}_{j}+\boldsymbol{\sigma}_{k} ; k=1,2, \ldots, K
$$

Where $\mathbf{x}_{k} \in \mathrm{C}^{N_{k} \times 1}$ is the $k$ th user's transmitted symbol, $\mathbf{H}_{k} \in \mathrm{C}^{N_{k} \times M}$ is the channel gain matrix from base station to the $k$ th user, $\mathbf{W}_{k} \in \mathrm{C}^{M \times N_{k}}$ is the precoding matrix of the $k$ th user, $\sigma_{k} \in \mathrm{C}^{N_{k} \times 1}$ is the additive white Gaussian 
noise(AWGN) at the $k$ th user. And normalized the transmit power.

\section{B. Sum Rate Calculation}

From (9) we can get the SINR (Signal Interference Noise Ratio) of user $k$

$$
\operatorname{SINR}_{k}=\frac{\left\|\mathbf{H}_{k} \mathbf{W}_{k}\right\|^{2}}{\boldsymbol{\sigma}_{k}^{2}+\sum_{j=1, j \neq k}^{K}\left\|\mathbf{H}_{k} \mathbf{W}_{j}\right\|^{2}}
$$

We use the precoding scheme based on the combination of BD and SLNR maximization. When the number of terminal antenna is not one, use BD precoding algorithm. Due to $\mathrm{BD}$ precoding can completely eliminate the interuser interference, the denominator of (10) is only the noise $\boldsymbol{\sigma}_{k}^{2}$. The energy of user $k$ in the numerator of (10) should be multiplied by the receiver equalizer matrix, then (10) will be

$$
\operatorname{SINR}_{k}=\frac{\left\|\mathbf{V}_{k}^{(1)} \mathbf{H}_{k} \mathbf{W}_{k}\right\|^{2}}{\boldsymbol{\sigma}_{k}^{\mathbf{2}}}
$$

Transmission rate of user $k$ is

$$
\mathrm{C}_{k}=\log 2\left(1+\operatorname{SINR}_{k}\right)
$$

For the terminal equipped with single antenna, we use the maximum SLNR precoding scheme and calculate the precoding matrix according to (8). Then we can calculate the SINR and transmission rate according to (10) and (12) respectively.

The sum rate of system can be described as follows.

$$
\mathrm{C}=\sum_{k=1}^{K} \mathrm{C}_{k}
$$

\section{Multi-user selection algorithm}

We increase one more user each time. First, we select the user whose channel quality is the best. In the following user selection process, we detect the terminal antenna, using maximum SLNR and BD precoding for single and multiple respectively. Then calculate the sum rate after adding the user, polling all of the users to select which has the largest sum rate.

However users equipped with multi-antenna will be dominant than single antenna users in the transmission rate performance. The probability selected multi-antenna users will increase when using the above selection method. At the same time selecting multi-antenna users will reduce the number a system can access simultaneously and lower the multi-user diversity. To solve this problem, we artificially add a value $\beta$ behind the sum-rate of single-antenna users, select the user and then calculate the real sum-rate after adding it. Value of $\beta$ determined through the simulation.

The selection algorithm is as follows.

step 1) Initialization: $\mathrm{T}=\{1, \ldots, N\} \quad, \quad S_{0}=\varnothing$, $\mathrm{C}_{\text {temp }}=0, i=1$.

step 2) When $i=1, \forall k \in \mathrm{T}$, calculate the Frobenius norm of the channel matrix $\left\|\mathbf{H}_{k}\right\|_{\mathrm{F}}^{2}$.Then $\pi(1)=\arg \max _{k \in \mathrm{T}}\left\|\mathbf{H}_{k}\right\|_{\mathrm{F}}^{2}$, calculate the rate of first user $\mathrm{C}_{1}, \mathrm{C}_{\text {temp }}=\mathrm{C}_{1}, \mathrm{~S}_{0}=\{\pi(1)\}$, $\mathrm{T}=\mathrm{T}-\{\pi(1)\}, i=i+1$.

step 3) When $i \geq 2, \forall k \in \mathrm{T}$, let $\mathrm{S}=\mathrm{S}_{0} \cup\{k\}$. Then detect the number of each user's antenna:

a) If the antenna is only one, according to (8), (10) and (12) calculate the precoding matrix, SINR, and transmission rate respectively;

b) If the antenna is not one, according to (5), (11) and (12) calculate the precoding matrix, SINR, and transmission rate respectively.

c) Then from (13) we can calculate the sum-rate $\mathrm{C}_{k}$ after adding user $k$.

step 4) We should detect the antenna of the $k$ th user:

a) If the $k$ th user has single antenna, let $\mathrm{C}_{k}=\mathrm{C}_{k}+\beta$.

b) $\pi(i)=\arg \max _{k \in \mathrm{T}} \mathrm{C}_{k}$, if the antenna of user $i$ is single, $\mathrm{C}_{i}=\mathrm{C}_{i}-\beta$.

step 5) If $\mathrm{C}_{i}>\mathrm{C}_{\text {temp }}$ and the antennas of all users are not greater than the number of base station side, update: $\quad \mathrm{C}_{\text {temp }}=\mathrm{C}_{i}, \quad \mathrm{~S}_{0}=\mathrm{S}_{0} \cup\{\pi(i)\}$, $\mathrm{T}=\mathrm{T}-\{\pi(i)\}, i=i+1$, back to Step 3 to continue. Else end. 


\section{SimULATION RESUlTS}

In Fig. 1, we plot the sum rate of users under the proposed user selection scheme, as a function of $\beta$ for $M=8$, the user randomly generates one or two antennas, $N=50$, normalized the transmit power, and SNR in the range of $0-20 \mathrm{~dB}$. We can see that the sum-rate performance will be the best while $\beta=1.4$. So in the next simulation we let $\beta=1.4$.

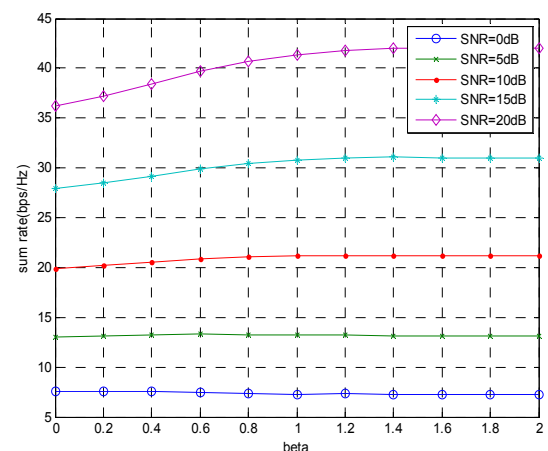

Fig 1. Sum rate of proposed user selection scheme with $M=8$, the user randomly generates one or two antennas, $N=50$, normalized the transmit power, and SNR in the range of $0-20 \mathrm{~dB}$ as a function of $\beta$.Optimal choice of $\beta$ is 1.4.

In Fig.2, we compare the sum-rate performance of the proposed strategy and BD strategy. The plot is obtained by averaging over 1000 independent times using the optimal $\beta$ values. $M=8$, the user randomly generates one or two antennas, $N=50$, normalized the transmit power. And $\mathrm{SNR}=[0: 2: 20]$. Fig.2 shows that the performance of the proposed scheme is improved significantly when SNR over $5 \mathrm{~dB}$. There has $30 \%$ performance improvement when SNR reaches $20 \mathrm{~dB}$. In the low SNR case, the performance is as well as the BD scheme. Because in the case of low SNR, the impact caused by additive white noise is primary. The performance loss caused by using BD algorithm to eliminate the interference in the terminal of the single antenna is relatively small. The interference between users is the main impact of the system in the case of high SNR. The disadvantage of BD precoding in the single antenna is obviously. Therefore, the proposed algorithm performance is much better than BD precoding in the high SNR case.

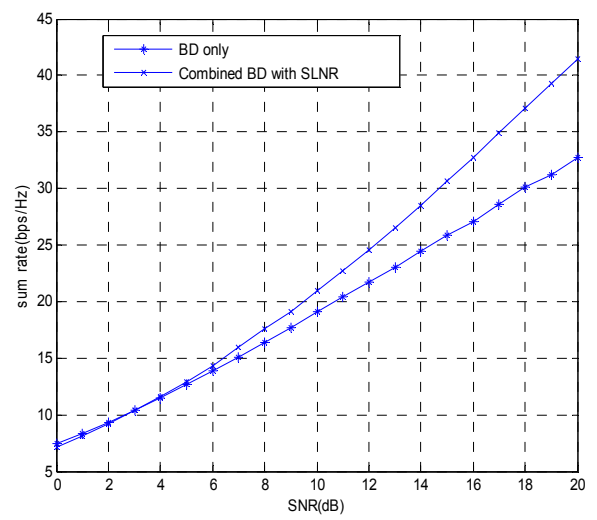

Fig 2. Sum-rate performance comparison of BD and combination of BD and SLNR. $M=8$, the user randomly generates one or two antennas, $N=50$, normalized the transmit power. And $\mathrm{SNR}=[0: 2: 20]$, using the optimal $\beta$.

\section{CONCLUSION}

With the development of science and technology, the scenario that one terminal configures more than one antennas will be increasingly popularity, while the single antenna users will exist in the system at the same time. There will be inconsistency of terminal antennas for quite a long period. The former user selection algorithm does not take this into account. The proposed algorithm combines the single-antenna users and multi-antenna users to precode separately and overcome the performance loss in the single antenna in case of BD precoding. In order to compensate the single-antenna users, we add value $\beta$ in the selection process. And ultimately the proposed algorithm has made great performance improvements.

\section{ACKNOWLEDGEMENT}

This paper is supported by NSFC (61201134, 61201135), the Fundamental Research Funds for the Central Universities (72124669) and the 111 Project(B08038).

\section{REFERENCES}

[1] Telatar E, "Capacity of multi-antenna Gaussian channels," European Trans. on Telecommun., 1999, 10(6): 585-595.

[2] Gesbert D, Kountouris M, Heath R W, Chae C B, Salzer T, "From single user to multiuser communications: shifting the MIMO paradigm," IEEE Signal Processing Magazine, 2007, 24(5): 36-46

[3] Vishwanath S, Jindal N, and Glodsmith A, "Duality, achievable rates, and sum-rate capacity of gaussian MIMO broadcast channels," IEEE Trans. On Information Theory, 2003, 49(10):2658-2668.

[4] M. Sharif and B. Hassibi, "A comparison of time-sharing, DPC, and beamforming for MIMO broadcast channels with many users," IEEE Trans. Commun., submitted for publication. 
[5] H. Weingarten, Y. Steinberg, and S. Shamai, "The capacity region of the Gaussian MIMO broadcast channel,” in Proc. IEEE Int. Symp. Inf. Theory (ISIT), Chicago, IL, 2004.

[6] Chen Xiao-han, Liu Ju, Zheng Li-na, Xu Hong-ji, “A Suboptimal User Selection Algorithm Based on Block Diagonalization and Fairness," JOURNAL OF ELECTRONICS \& INFORMATION TECHNOLOGY. 2009, 31(7).

[7] LV Jing, LUO Hanwen, and ZHANG Jing, "A New User Selection Algorithm for MIMO System with Block Diagonalization," Journal of Shanghai Jiaotong University. 2010, Vol. 44.

[8] V.Stankovic, M.Haardt, and M.Fuchs, "Combination of block diagonalization and THP transmit filtering for downlink beamforming in multi-user MIMO systems," in Proceedings of the European Conference on Wireless Technology (ECWT 2004), Amsterdam, The Netherlands, October 2004, to be published.

[9] Q.Spencer and M.Haardt, "Capacity and downlink transmission algorithms for a multi-user MIMO channel," in Proc.36th Asilomar Conf. on Signals, Systems, and Computers, Pacific Grove, CA IEEE Computer Society Press, November 2002.

[10] LI Ying-dong, ZHU Guang-xi, “Joint User antenna Power Allocation Algorithm Based on SLNR for Multi-user MIMO Downlink Channels," JOURNAL OF CHINESE COMPUTER SYSTEMS, 2010, 31(9).

[11] Cao Ying-hong, Yin Fu-liang, Chen Zhe, "Downlink Precoding for Multi-user Quasi-orthogonal Space Time Block Coded MIMO System," JOURNAL OF ELECTRONICS \& INFORMATION TECHNOLOGY, 2009, 31(7).

[12] M.Sadek, A.Tarighat, and A.H.Sayed, "A Leakage-based Precoding Scheme for Downlink Multi-user MIMO Channels," IEEE Trans. On Wireless Communications, vol.6, 2007, PP.1711-1721. 